\title{
Statistical Study of nCoV-SARS-2 Disorder in Nigeria Social Dynamics using Correlation and Gravity Law
}

\author{
Ibrahim Abubakar Sadiq ${ }^{1}$ \\ Nazir Muhammad Isah ${ }^{2}$ \\ ${ }^{1,2}$ Department of Statistics, Ahmadu Bello University, Zaria, Nigeria. \\ ${ }^{1}$ Department of Mathematics and Statistics, Mewar University Chittorgarh, Rajasthan, India.
}

\begin{abstract}
In this study, the populated and production hub Lagos state is compared with the rest of Nigerian thirty-seven states including the federal capital territory (FCT Abuja), and their concerned distance was used to find the gravitational attraction by applying the gravity model in social dynamics. We have applied the correlation analysis to find a relationship between gravitational attraction during the COVID-19 outbreak from the epicenter state and the number of disease cases in the rest of the states (ROS). We have found out, gravity law in social dynamics has no dependence on the COVID-19 outbreak and it's widespread in Nigeria from the epicenter state. We also discovered that the inverse square law has no reliance on the number of COVID-19 cases in terms of gravitational attraction.
\end{abstract}

Keywords: Statistics, Correlation, Gravitational Attraction, Inverse Square Law, Social Dynamics, COVID-19

\section{INTRODUCTION}

Severe acute respiratory syndrome 2 is the transmittable virus that originated from the recently uncovered coronavirus. This novel infection and its syndrome remained unidentified earlier the epidemic which commenced in Wuhan, China, in late 2019. Coronavirus currently remains a global challenge infecting most nations increasingly. The first index case of the Coronavirus pandemic in Nigeria was recorded on 27th February 2020 an Italian citizen in Lagos tested positive for the virus. As of 12th December 2020, the Nigeria Centre for Disease Control (NCDC) has informed about an aggregate of 72140 cases in which 65722 were recovered and 1190 deaths in the country [1].

In high cases of the disease, the epicenter in this region where the most infections occurs becomes a place of study [5]. Nigeria's most commercial center has arisen as the epicenter of the country's COVID-19 pandemic as the town a lone records for more than a third of the total case count. The rambling city of Lagos, amongst Nigeria's most congested, is battling against this curve of Covid-19 virus that has traversed one lakh in Nigeria's second-most populated state [6]. Gravity law attracts all other objects in this space with a force that is relative to the resultant of their masses and inversely related to the square of the separation between their centers. This is a universal physical law generated from experimental interpretations by what Isaac Newton called inductive rational. It is a portion of old mechanics and was expressed in Newton's work Philosophia Mathematica ("the Principia"), first printed on $5^{\text {th }}$ July 1689 [7].

\section{METHOD}

For centuries, social scientists have been applying a reformed version of Isaac Newton's Law of Gravitation to forecast the movement of people, information, and commodities between states and even nations. Experts of Social sciences described the gravity model, reformed law of gravitation, proceeds into the version that, the population magnitude of two dwellings and their separation. Since bigger spaces attract persons, better than minor spaces and spaces nearer composed to have a much attraction, the gravity model integrates these dual features. Essentially, the gravity model is applied to justify every interaction or movement that is anticipated to flow from one space to another. The comparative power of a connection between two states is computed by multiplying the magnitude of the population of the epicenter state by the magnitude of the population of the rest of the states (ROS) each and then dividing the amount by the separation within the two states squared [8].

\subsection{The Gravity Model}

$P_{x y}=\frac{Q_{x} R_{y}}{\phi_{x y}^{2}}$

Where $P_{x y}$ is the gravitational attraction between Lagos and any one of the thirty-seven states in Nigeria including FCT Abuja, $Q_{x}$ is the population of the epicenter Lagos, $R_{y}$ is the population of each of the remaining states, and $\phi^{2}$ is the distance between epicenter and the rest of the state each. 


\subsection{Correlation Analysis}

The Product Moment Coefficient of Correlation is the amount of the degree of a steady relationship between two variables and is represented by $\rho$. The PMCC indicates a line of best fit using the numerical observations of dual variables, and the CCMP shows how distant all these data points are to this line of best fit [8].

The PMCC has a range of values from -1 to +1 . A reading zero shows that there is no relationship between the two variables. A value above zero shows a positive relationship; that is to say, when one variable rises, the other variable also increases. A value below zero shows a negative relationship indicates if the value of one variable decreases, the value of the other variable increases, and vice versa [8]. This is depicted in the below figures:
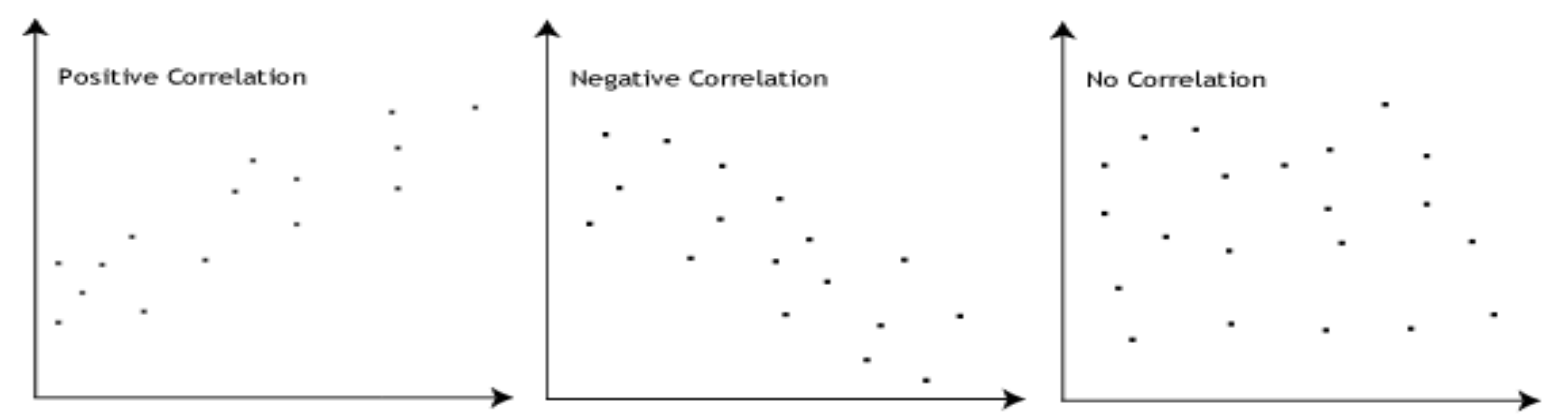

The stronger the association of the two variables, the closer the PMCC $\rho$, resolve to be either positive or negative one (+1 or -1$)$ subject on whether the association is $+\mathrm{VE}$ or $-\mathrm{VE}$, correspondingly. Achieving a value of positive or negative one indicates that all our numerical observational points are captured on the line of best fit, there are no numerical data points that indicate any disparity away from the line. A value for $\rho$ within +1 and -1 , for instance, $\rho=0.800$ or -0.400 shows that there is deviation round the line of best fit. The nearer the value $\rho$ to zero the higher the deviations within the line of best fit [8]. Diverse associations and their PMCC are depicted in the below figure:

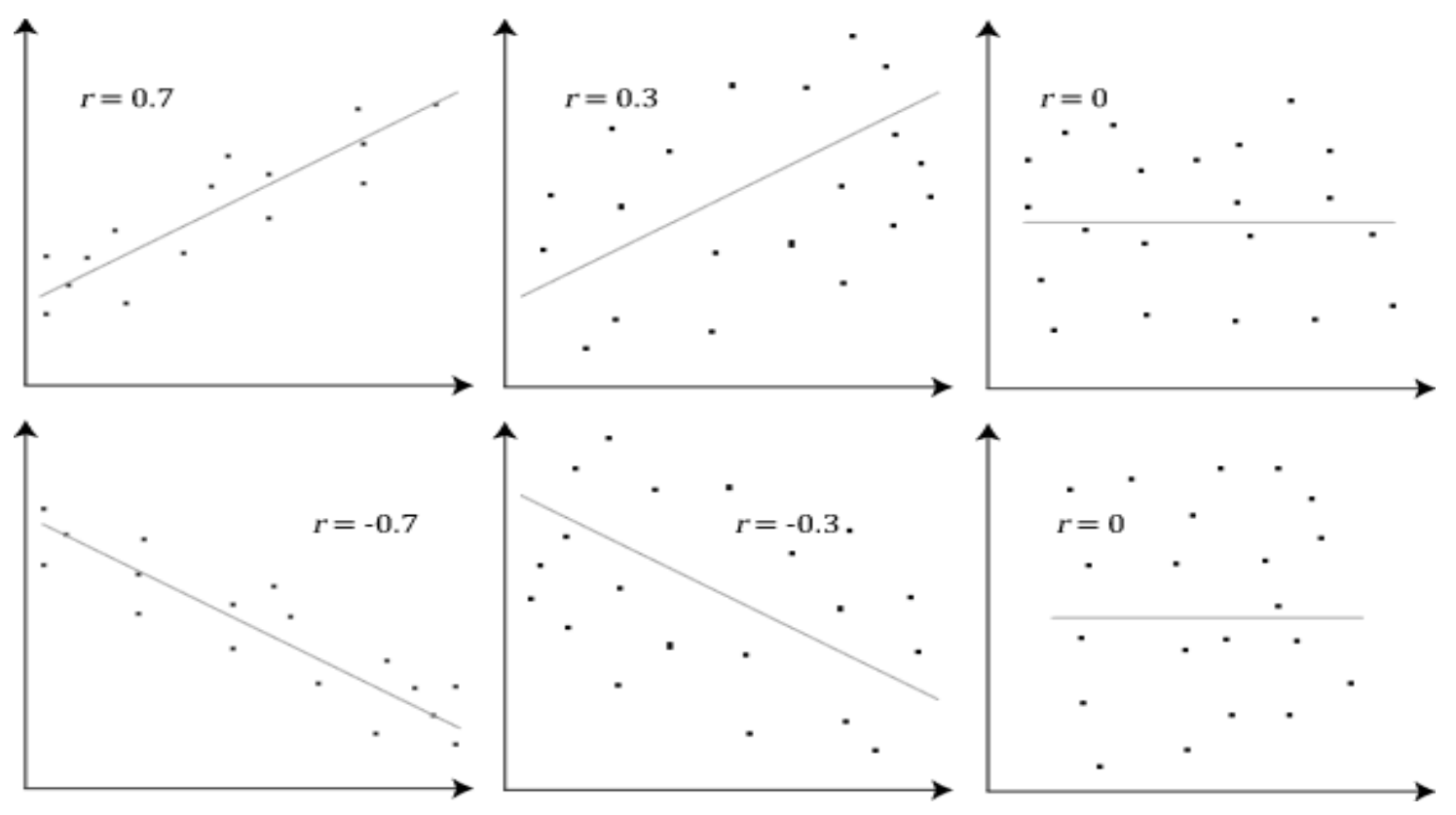

\subsection{The Single Factor Analysis of variance}

The Analysis of variance, ANOVA is a technique of inferential statistical method applied to measure the equivalence of three, four, or more population or sample averages.

$\mathrm{H}_{0}: \mu_{1}=\mu_{2}=\mu_{3}=\ldots=\mu_{\mathrm{k}}$

This technique can also be stated as one-way ANOVA since it involves the application of a single, principle, characteristic or property, for classifying the samples or populations. This specific property is times stated as a factor or treatment. A factor (or treatment) is a characteristic that permits us to differentiate the variety of populations from one collection to another. The main purposes of a single factor analysis of variance are as follows [9]. 
i.To estimate treatment means,

ii.To estimate the differences in treatment means

iii.To test hypotheses for statistical significance

Table 1 is a layout for shortening the major results in a single-factor analysis of variance computations. The next single factor analysis of variance (ANOVA) table demonstrates the necessary calculations and having associations among the different analysis of variance table components.

Table1. Single Factor analysis of ANOVA table

\begin{tabular}{|c|c|c|c|c|}
\hline Source of Variation & Degree of Freedom & Sum of Squares (SS) & Mean Sum of Squares (MSS) & F-Test \\
\hline Treatment & $\mathrm{k}-1$ & $S S_{\text {treatment }}$ & $M S_{\text {treatment }}=\frac{S S_{\text {treatment }}}{k-1}$ & $F=\frac{M S_{\text {treatment }}}{M S_{\text {error }}}$ \\
\hline Error & $\mathrm{N}-\mathrm{k}$ & $S S_{\text {error }}$ & $M S_{\text {error }}=\frac{S S_{\text {error }}}{N-k}$ & \\
\hline Total & $\mathrm{N}-1$ & $S S_{\text {total }}$ & & \\
\hline
\end{tabular}

The squares sum with the association of

$$
\begin{aligned}
& S S_{\text {total }}=S S_{\text {treatment }}+S S_{\text {error }} \\
& \text { And } S S_{\text {total }}=\sum_{i=1}^{k} \sum_{j=1}^{n}\left(x_{i j}-\bar{x}\right)^{2}, S S_{\text {treatment }}=\sum_{i=1}^{k} n_{i}\left(\overline{x_{i}}-\bar{x}\right)^{2}, S S_{\text {error }}=\sum_{i=1}^{k} \sum_{j=1}^{n}\left(x_{i j}-\overline{x_{i}}\right)^{2}
\end{aligned}
$$

The DF (degrees of freedom) also shares a similar association:

$$
d f\left(S S_{\text {total }}\right)=d f\left(S S_{\text {treatment }}\right)+d f\left(S S_{\text {error }}\right)
$$

The Squares Sum of Mean for the error and treatment are computed by dividing the SS (Sums of Squares) by the DF (degrees of freedom) for each. While SS is additive, the MSS (Mean Sums of Squares) are not. The F-ratio statistic is calculated by dividing the $M S_{\text {treatment }}$ by the $M S_{\text {error }}$. The $M S_{\text {treatment }}$ is the $\mathrm{S}_{\mathrm{B} 2}$ and the $M S_{\text {error }}$ is the $\mathrm{S}_{\mathrm{W} 2}[10]$.

$F=\frac{S_{B 2}}{S_{w 2}}=\frac{M S_{\text {total }}}{M S_{\text {error }}}$

\section{DISCUSSION OF RESULTS}

We have studied the application of gravity law in social dynamics using the gravity model to find the dependence of COVID-19 spread from the pandemic epicenter Lagos state in Nigeria and the rest of the thirty-seven states including FCT Abuja where affected by this outbreak. The data were examined by using a Google map to obtain the actual distance from Lagos as our COVID19 outbreak epicenter in connection to the rest of the states. The population figures of each state were deeply examined which allowed us to compute the gravitational attraction. $P_{x y}$, the gravitational attraction between Lagos and any one of the thirty-seven states including FCT Abuja was computed by multiplying $Q_{x}$ the population of the epicenter Lagos and $R_{y}$ as the population of each of the remaining states and dividing by $\phi_{x y}^{2}$ the separation from the epicenter and the rest of the state each. 
Table 1: Descriptive Statistics Results

\begin{tabular}{|c|c|c|c|c|}
\hline \multirow{3}{*}{ Table } & \multirow{2}{*}{\multicolumn{2}{|c|}{ escriptive Statistics Results }} & \multirow{2}{*}{$\begin{array}{r}\text { Statistic } \\
1949.7297 \\
\end{array}$} & \multirow{2}{*}{$\begin{array}{r}\text { Std. Error } \\
682.18036\end{array}$} \\
\hline & & & & \\
\hline & 95\% Confidence Interval for Mean & Lower Bound & 566.2038 & \\
\hline & & Upper Bound & 3333.2556 & \\
\hline & 5\% Trimmed Mean & & 1216.8288 & \\
\hline & Median & & 802.0000 & \\
\hline & Variance & & 17218691.814 & \\
\hline & Std. Deviation & & 4149.54116 & \\
\hline & Minimum & & 5.00 & \\
\hline & Maximum & & 24614.00 & \\
\hline & Range & & 24609.00 & \\
\hline & Interquartile Range & & 1553.00 & \\
\hline & Skewness & & 4.856 & .388 \\
\hline & Kurtosis & & 26.126 & .759 \\
\hline \multirow[t]{14}{*}{ Gravitational Attraction } & Mean & & 481855322.7835 & 243900038.43152 \\
\hline & 95\% Confidence Interval for Mean & Lower Bound & -12796881.9983 & \\
\hline & & Upper Bound & 976507527.5653 & \\
\hline & 5\% Trimmed Mean & & 201790751.6334 & \\
\hline & Median & & 123137616.9000 & \\
\hline & Variance & & 2201027463635123 & \\
\hline & & & 710.000 & \\
\hline & Std. Deviation & & 1483586014.90952 & \\
\hline & Minimum & & .00 & \\
\hline & Maximum & & 8845687264.00 & \\
\hline & Range & & 8845687264.00 & \\
\hline & Interquartile Range & & 215790051.54 & \\
\hline & Skewness & & 5.339 & .388 \\
\hline & Kurtosis & & 30.038 & .759 \\
\hline
\end{tabular}




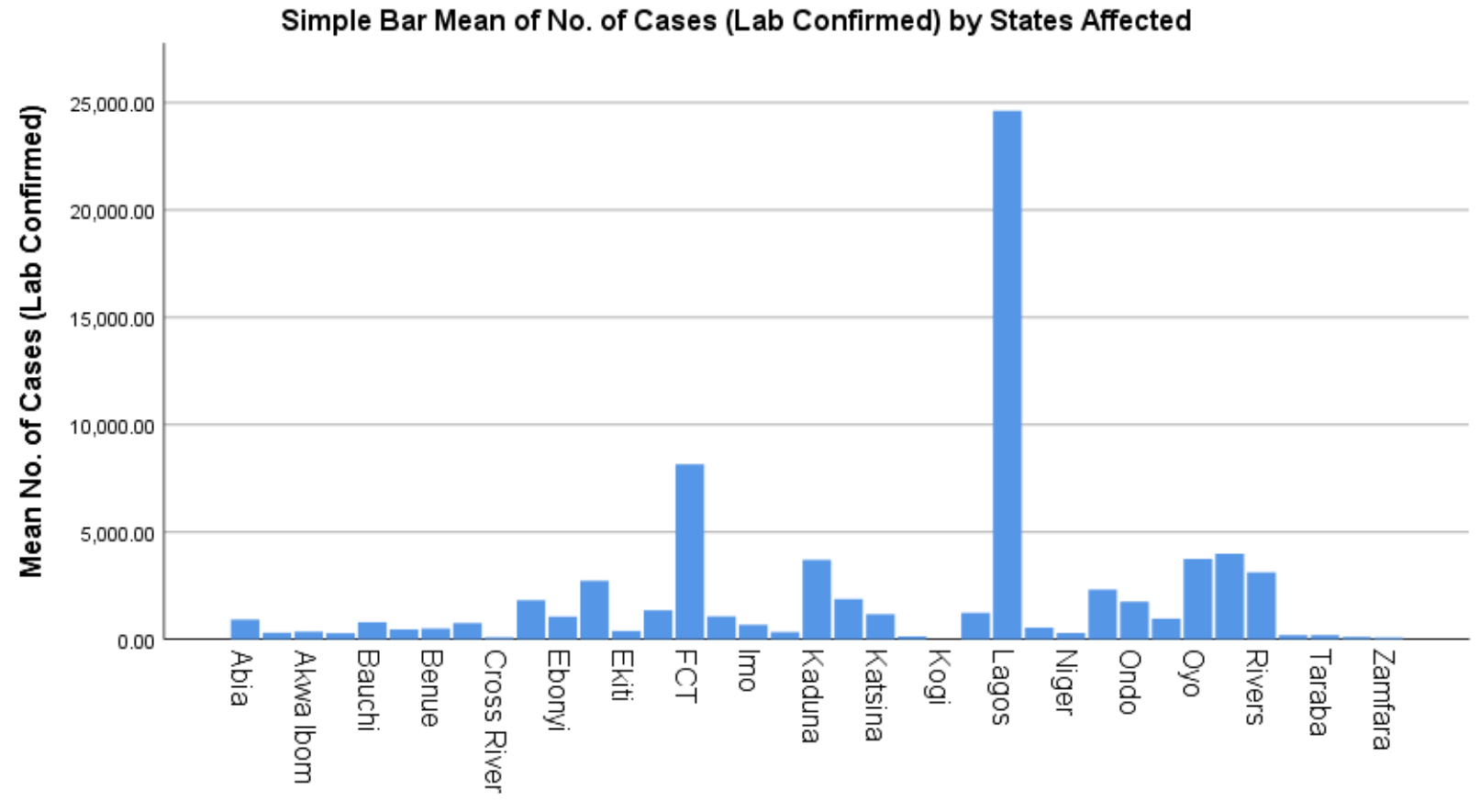

States Affected

Fig 1.Bar Chart of Covid-19 cases per the state

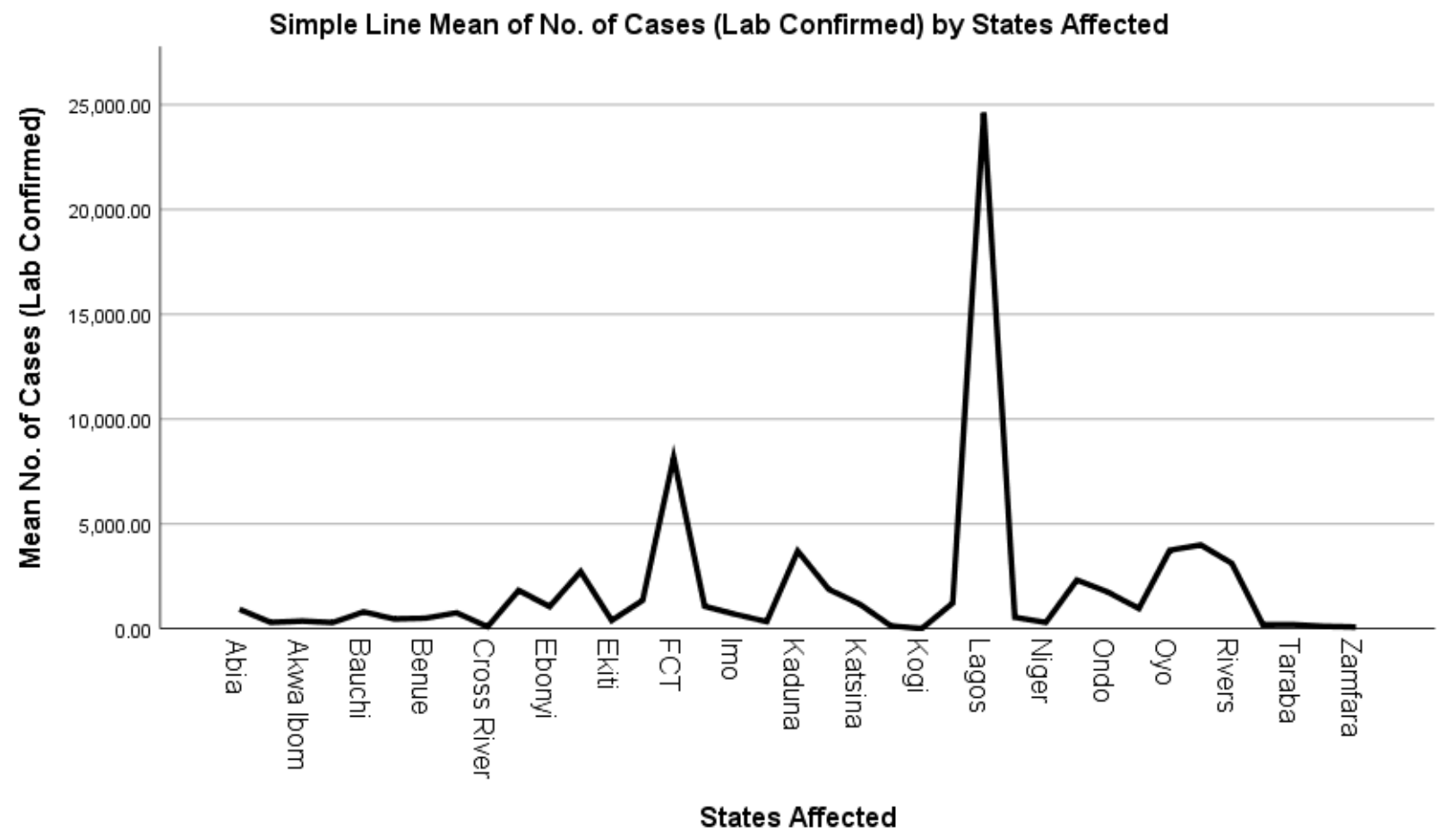

Fig 2. Line Plots of Covid-19 cases per State 


\subsection{Result 1}

Figures 1 and 2 displayed the numerical digit of COVID-19 cases per state. Lagos is the state that recorded the largest cases, examined in our study as the epicenter of the COVID-19 outbreak. The study also shows that FCT Abuja recorded the second high number of cases, whereas Osun, Oyo, Rivers, and Kaduna are the third states with a relatively high number. The remaining state has reported a very low number of outbreak cases.

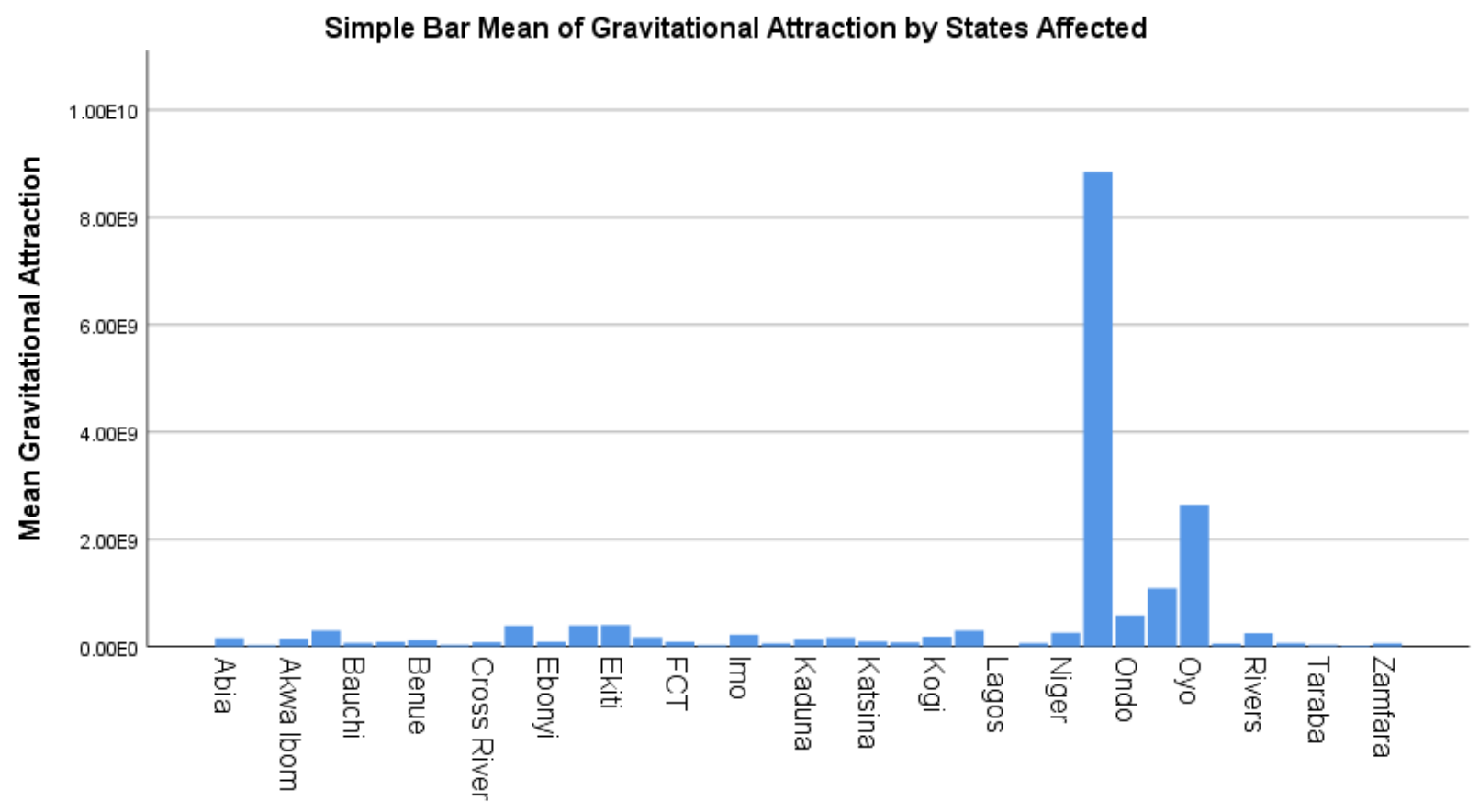

States Affected

Fig 2. Bar Chart of Gravitational Attraction per State from Epicenter

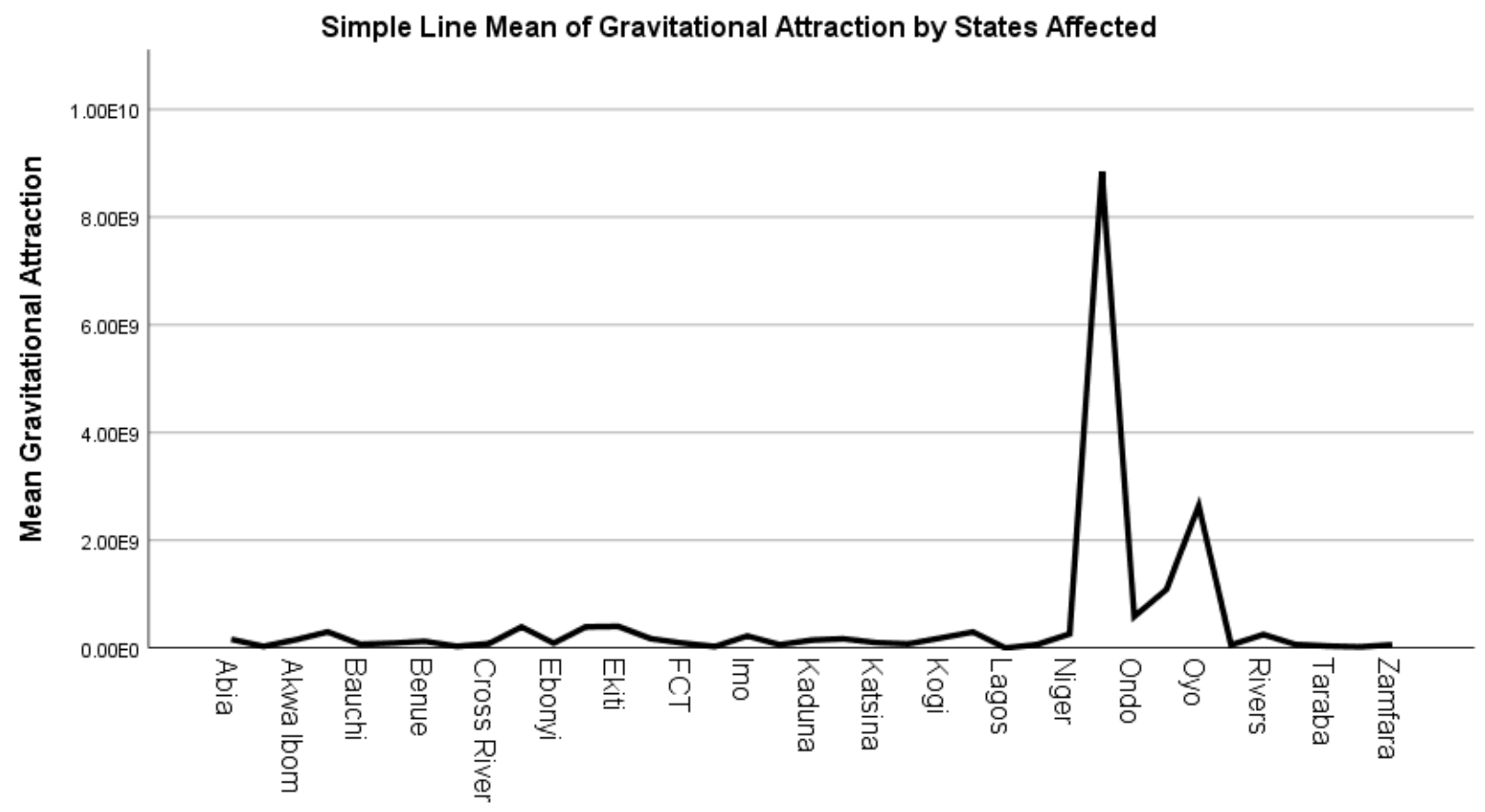

States Affected

Fig 2. Line Plot of Gravitational Attraction per State from Epicenter 


\subsection{Result 2}

Figure 3 and 4 indicates the gravitational attraction in social dynamics computed using a gravity model. It shows that states which are very close to the epicenter state Lagos have a higher value of gravitational attraction compared to those far away states since gravitational attraction takes an inverse relation with separation according to gravity law. Our first findings depict that states with high gravitational attraction have many social dynamics affected such as movement, commercial activities, transportation, festivities, and other social interactions but the numerical digit of COVID-19 cases has no correlation with gravitational attraction. A state like Ogun, Oyo, Osun, Ondo, and Ekiti was mostly affected in terms of their social activities relative to the pandemic epicenter whereas a state like Borno, Gombe, Yobe, Adamawa, Taraba, Bauchi, etc. are less affected.

Table 2

Pearson's product-moment correlation

\begin{tabular}{ll|r|r} 
& & No. of Cases (Lab & Gravitational \\
& Confirmed) & Attraction \\
\hline No. of Cases (Lab Confirmed) & Pearson Correlation & 1 & .018 \\
\cline { 2 - 4 } & Sig. (2-tailed) & 37 & .917 \\
\cline { 2 - 4 } Gravitational Attraction & $\mathrm{N}$ & .018 & 37 \\
\hline & Pearson Correlation & .917 & 1 \\
\hline & Sig. (2-tailed) & 37 & 37 \\
\hline
\end{tabular}

Alternative hypothesis: true correlation is not equal to zero.

\subsection{Result 3}

The statistical analysis was examined by the use of R-programming, Gretl, and SPSS software. Table 1 is the descriptive measur es of our study's variables. Table 2 depicts Pearson's product-moment correlation result. The result of the estimated correlation $\mathrm{b}$ etween gravitational attraction and COVID-19 concerning our outbreak epicenter is 0.018 . This value revealed statistically that C OVID-19 has a very weak relationship with gravitational attraction with connection to gravity law in social dynamics of epicent er state and the ROS. The test of significance of the correlation value by applying T-test statistics to the P-value (0.917) at a 0.05 alpha level of confidence was forced the rejection of our null hypothesis. In the case of the Alternative hypothesis, true correlati on is not equal to zero. The P-value is less than and closer to the critical region indicate additional evidence for non-rejection of our alternative hypothesis.

\section{CONCLUSION}

To conclude, the gravity law in social dynamics has no dependency on the COVID-19 outbreak and its spread in Nigeria from the epicenter state of Lagos and the rest of the state nationwide. We discovered that the separation of inverse square law applied has no reliance on the numerical digit of COVID-19 cases against gravitational attraction. As per the instruction of the medical committee and our findings indicates that the measures taking by the government such as movement restrictions and advice from the health professionals highly contributed to the non-dependence of the pandemic outbreak to gravity law in social dynamics. The outbreak of the pandemic is still on the rising stage in Nigeria. This study may be of tremendous benefit to the government, WHO, research scholars, security agencies, commercial institutions, religious centers, and many more in bringing to an end this deadly virus.

\section{REFERENCES}

[1] https://www.who.int/emergencies/diseases/novel-coronavirus-2019/question-and-answers-hub

[2] "Home/NCDC/GO/"moh.gov.ng Retrieved 23 $23^{\text {rd }}$ May 2020.

[3] "Nigeria's case count crosses 20,000, Abuja eases restriction: Covid-19 news today" Daily Trust. 19 ${ }^{\text {th }}$ May 2020. Retrieved $20^{\text {th }}$ May 2020.

[4] "Lagos confirmed first novel coronavirus case in Nigeria". Daily Trust today. $27^{\text {th }}$ February 2020.

[5] Fuis, Gary; Wald, Lisa. "RS-Central Alaska. The Denali Fault Earthquake of 2002". USGS. Retrieved 2008-04-20.

[6] https://www.bloombergquint.com/coronavirus-outbreak/in-charts-how-Lagos-emerged-as-the-epicenter-of-covid-19-in-Nigeria.

[7] I Newton: "In experimental philosophy particular propositions are inferred from the phenomena and afterward rendered general by induction": "Principia", Book 3, General Scholium, at p.392 in Volume 2 of Andrew Motte's English translation published 1729.

[8] https://www.thoughtco.com/what-is-the-gravity-model-4088877

[9] https://statistics.laerd.com/statistical-guides/pearson-correlation-coefficient-statistical-guide.php

[10] https://courses.lumenlearning.com/suny-natural-resourcesbiometrics/chapter/chapter-5-one-way-analysis-of-variance/ 